\title{
Reduction of Effects of Noise on the Inverse Problem of Electrocardiography with Bayesian Estimation
}

\author{
Y Serinagaoglu Dogrusoz ${ }^{1}, \mathrm{~L}$ R Bear ${ }^{2}, \mathrm{~J} \mathrm{Svehlikova}^{3}, \mathrm{~J} \mathrm{Coll-Font}^{4}$, W Good $^{5}, \mathrm{R}_{\text {Dubois }}^{2}$, E van

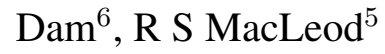 \\ ${ }^{1}$ Electrical and Electronics Engineering Department, METU, Ankara, Turkey \\ ${ }^{2}$ IHU-LIRYC, Université de Bordeaux, Bordeaux, France \\ ${ }^{3}$ Institute of Measurement Science, Slovak Academy of Sciences, Bratislava, Slovakia \\ ${ }^{4}$ Radiology Department at Boston Children's Hospital, Boston (MA), USA \\ ${ }^{5}$ Dept. of Bioengineering and SCI Institute, University of Utah, Salt Lake City (UT), USA \\ ${ }^{6}$ Peacs BV, Nieuwerbrug aan den Rijn, The Netherlands
}

\begin{abstract}
To overcome the ill-posed nature of the inverse problem of electrocardiography (ECG) and stabilize the solutions, regularization is used. Despite several studies on noise, effect of prefiltering of ECG signals on the regularized inverse solutions has not been explored. We used Bayesian estimation for solving the inverse ECG problem with and without applying various prefiltering methods, and evaluated our results using experimental data that came from a Langendorff-perfused pig heart suspended in a humanshaped torso-tank. Epicardial electrograms were recorded during $R V$ pacing using a 108-electrode array, simultaneously with ECGs from 128 electrodes embedded in the tank surface. Leave-one-beat-out protocol was used to obtain the prior probability density function ( $p d f)$ of electrograms and noise statistics. Noise pdf was assumed to be zero mean-Gaussian, with covariance assumptions: a) independent and identically distributed (noi-iid), b) correlated (noi-corr). Reconstructed electrograms and activation times were compared to those directly recorded by the sock for 3 beats selected from the recording. Noi-corr is superior to noi-iid when the training set is a good match to data, but for applications requiring activation time derivation, careful selection of preprocessing methods, in particular to adequately remove high-frequency noise, and an appropriate noise model is needed.
\end{abstract}

\section{Introduction}

Inverse problem of electrocardiography (ECG), also known as electrocardiographic imaging (ECGI), aims to reconstruct cardiac electric sources using measurements from the body surface (BSPM) obtained via densely placed electrodes, and a mathematical model describing the relationship between the sources and measurements [1]. However, due to attenuation and smoothing effects within the body, inverse ECG problem is ill-posed, and regularization should be applied to stabilize the solutions. Several techniques have been proposed in literature to overcome this ill-posedness, such as Tikhonov regularization [2], Bayesian estimation [3], Kalman filtering [4], spline fitting [5]. An important criteria in selecting an inverse solution method for a specific clinical application is its robustness to errors in the mathematical model, and noise in the ECG signals.

There has been ongoing interest among researchers to understand the effects of measurement noise [4] and model errors $[4,6]$ on the regularized inverse solutions. Most of these methods aim to reduce the effects of noise within the inverse solution method. [7] reported that anisotropic filters applied to BSPMs before solving the inverse problem yielded better reconstructions. However, despite these efforts, there is still need for a systematic study to assess and overcome the noise effects by applying prefiltering to the ECG signals before solving the inverse problem. To address this issue, 'Signal Processing Workgroup' was created during the meeting at Computing in Cardiology 2017. The work presented in this manuscript resulted from combined efforts of researchers in this workgroup.

Selected filters were applied to ECG signals in two stages, first stage aims to remove high frequency noise, including the $50 \mathrm{~Hz}$ line interference, and the second stage removes the base line drift. Epicardial electrograms were reconstructed using Bayesian Maximum A Posteriori (MAP) estimation, assuming jointly Gaussian epicardial and torso potentials, for raw (unfiltered), and filtered tank ECGs. Leave-one-beat-out protocol was used to obtain the prior probability density function (pdf) of electro- 


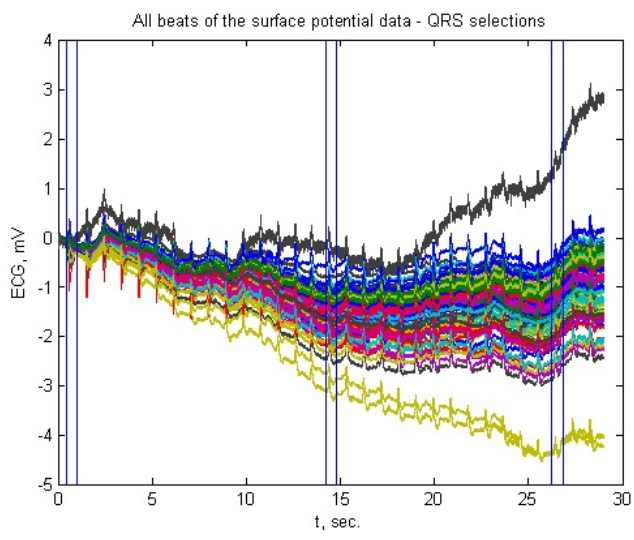

Beat 1

Beat 2

Beat 3

Figure 1. BSPMs recorded from 128 electrodes on the tank surface. Vertical lines show the three selected test beats for ECGI.

grams and noise statistics. Noise pdf was assumed to be zero-mean Gaussian. Two different noise covariance assumptions, independent and identically distributed noise, and correlated noise, were used to study the effects of noise model on the solutions with filtered and raw ECGs. Reconstructed and recorded electrograms and the corresponding activation time maps were compared qualitatively and quantitatively.

\section{Methods}

Experimental data came from a Langendorff-perfused pig heart suspended in a human-shaped torso-tank. Epicardial electrograms were recorded during RV pacing using a 108-electrode array, simultaneously with ECGs from 128 electrodes embedded in the tank surface. A more detailed explanation of the experimental procedure can be found in [8] and the references therein. Data consisted of $30 \mathrm{~min}-$ utes of ECG recordings, with 31 beats. ECG signals were highly contaminated by high frequency noise, $50 \mathrm{~Hz}$ line interference and baseline drift noise, as shown in Figure 1.

Forward problem relating the epicardial data to BSPMs was solved using the boundary element method (BEM) [9] assuming a homogeneous volume between the heart and torso surfaces. In this solution, epicardial and torso surfaces were represented by using meshes with 1012 nodes and 128 nodes, respectively.

Inverse problem was solved using Bayesian Maximum A Posteriori (MAP) estimation method explained in [3]. This is a statistical approach, in which the epicardial potentials and the noise are treated as random variables, or multivariate random vectors. Bayesian MAP estimation aims to obtain the posterior probability density function (pdf) of the epicardial potentials based on the likelihood function of the measurements (i.e., pdf of measurements given the epicardial potentials) and an a priori pdf of the epicardial potentials, which stands for the available prior information on the epicardial potentials. Solution is defined as the epicardial potential distribution that maximizes the posterior pdf. A detailed theoretical background on this method and its application to ECGI can be found in [3]. In this study, we assumed that the epicardial potentials and BSPMs are jointly Gaussian; prior pdf is a Gaussian distribution with a well-defined mean and covariance; noise in the measurements are zero-mean Gaussian and uncorrelated with the epicardial potentials.

Three out of 31 beats were chosen as the test beats, as shown in Figure 1, and inverse problem was solved only for these test beats. For each test beat, a training set was composed consisting of all the beats excluding the test beat itself (leave-one-beat-out protocol). Prior pdf and noise covariance were then obtained using the corresponding training set for each test beat, as described in [3]. Two different models were used to define the noise covariance; a) independent and identically distributed (noi-iid), b) correlated (noi-corr), which were also estimated using the training sets.

Preprocessing of ECG signals was carried out in two consecutive stages; stage 1 included 8 different highfrequency noise removal filters (Filt-High), and stage 2 consisted of 5 different baseline drift removal filters (BDR), as described in [10] in detail. Inverse problem was solved and compared for four different preprocessing scenarios: 1) no processing (the raw signal), 2) Filt-High only, 3) BDR only, and 4) Filt-High and BDR combined.

For each solution, reconstructed epicardial potentials were compared quantitatively with the sock recordings at the corresponding 108 nodes in terms of root mean square (RMS) voltage, root mean sqaured error (RMSE) and correlation coefficient (CC). Activation times were also calculated for the recorded and estimated epicardial potentials using the maximum derivative (dV/dt) as described in [11]. Activation times at 108 measurement locations were then compared using Pearson's Correlation. Statistical comparisons were also performed on these results using unpaired Student's t-tests with significance taken for $p>0.05$.

\section{Results}

Top panel in Figure 2 shows the RMS Voltage for recorded (black) and noi-corr (blue/cyan) and noi-iid (red/magenta) inverse solutions with and without stage 1 filtering (Filt-High), and bottom panel shows the mean RMS voltage for all preprocessing scenarios. For all these scenarios, noi-iid RMS voltage was significantly smaller than recorded values $(p<0.05)$. There was no significant difference between noi-corr RMS voltage and recorded 

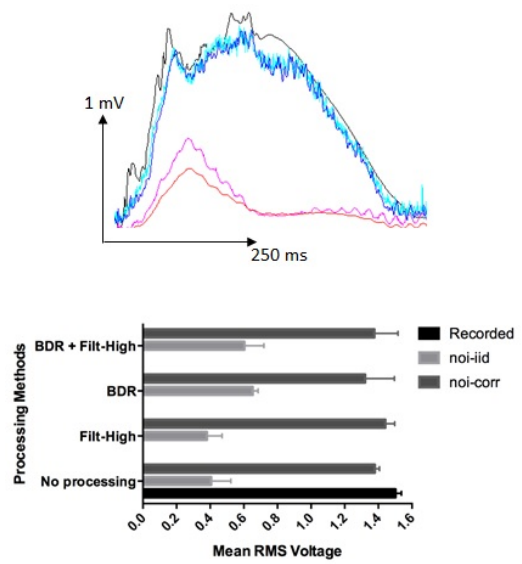

Figure 2. Top: RMS voltage for recorded (black) and noi-corr (blue/cyan) and noi-iid (red/magenta) inverse solutions with and without high pass filtering. Bottom: Mean RMS values for all preprocessing scenarios.

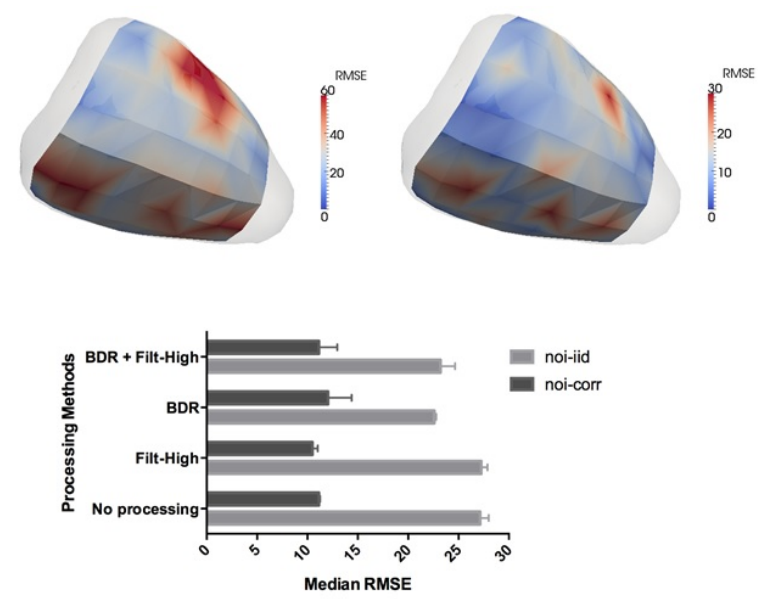

Figure 3. Top: RMSE at electrode locations for noi-iid (left) and noi-corr (right) without signal processing. Bottom: Median RMSE values for all preprocessing scenarios.

when signal processing was used $(p>0.07)$ but there was a significant difference when no processing was used $(p=0.009)$.

Top panel in Figure 3 shows the RMSE values at electrode locations for noi-iid (left) and noi-corr (right) without signal preprocessing, and bottom panel shows the median RMSE values for all preprocessing scenarios. For noiiid, RMSE values were significantly larger than noi-corr $(p<0.05)$. For noi-iid, applying BDR (with or without Filt-High) significantly improved RMSE $(p<0.05)$. For noi-corr, applying BDR significantly deteriorated results compared to Filt-High $(p<0.05)$.

Top panel in Figure 4 shows the CC values at electrode
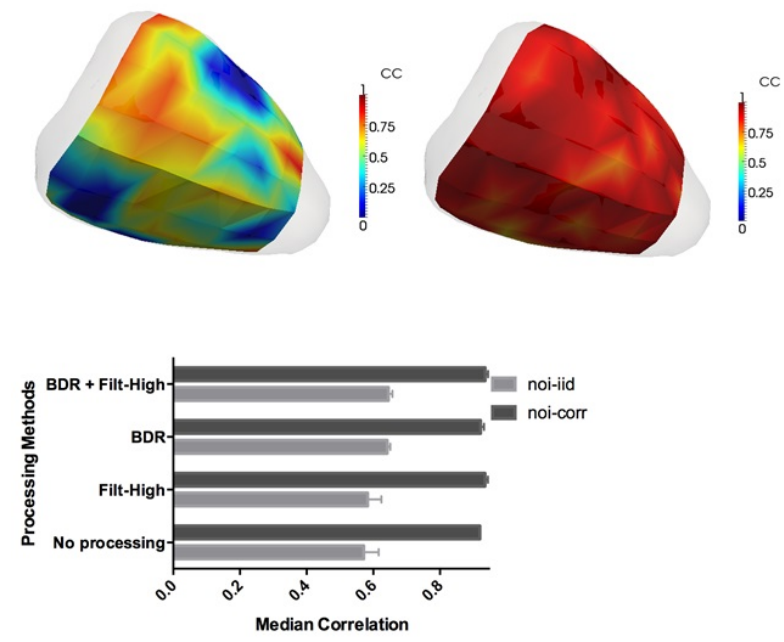

Figure 4. Top: CC at electrode locations for noi-iid (left) and noi-corr (right) without signal processing. Bottom: Median CC values for all preprocessing scenarios.

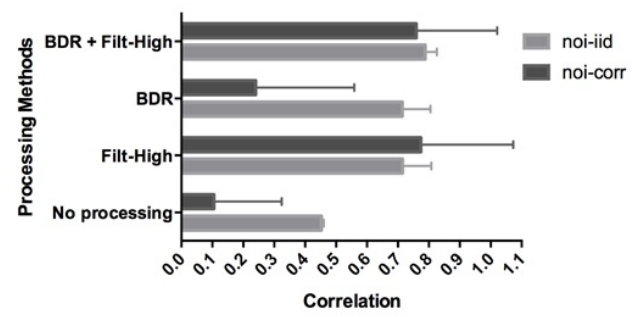

Figure 5. Correlation values of the activation times for all preprocessing scenarios.

locations for noi-iid (left) and noi-corr (right) without signal preprocessing, and bottom panel shows the median CC values for all preprocessing scenarios. For noi-iid, applying BDR (with or without Filt-High) significantly improved CC $(p<0.05)$ but not Filt-High alone. For noicorr, no processing method improved results $(p>0.05)$ though applying BDR significantly deteriorated results compared to BDR with Filt-High $(p<0.05)$.

Figure 5 shows the correlation values of the activation times for all preprocessing scenarios. For noi-iid, reconstructions after BDR and Filt-High combination were significantly better than using no processing $(p<0.05)$. For noi-corr, reconstructions were significantly better with Filt-High processing, when used with or without BDR $(p<0.05)$. There was no significant difference between noi-iid and noi-corr except when BDR alone was applied to signals $(p<0.001)$. 


\section{Discussions}

In this study, we approached measurement noise effects on ECGI from two aspects; preprocessing of the ECG signals, and modifying the statistical noise model.

Noi-corr is a more realistic noise model, which takes into account the spatial correlation of noise over the body surface. It produces better reconstructions when the noise in the measurements matches well with the training set properties. However, when the training set deviates from the measurements, reconstructions deteriorate, as demonstrated with the BDR results; when BDR is applied to the test beat but not to the training set, noi-corr results worsened. Noi-iid on the other hand is a simpler model, and only requires a single parameter (noise variance) to represent the noise statistics. It has the effect of reducing reconstructed electrogram amplitudes, but due to its simplicity, if there is no clear information on the noise model, it is superior to noi-corr, especially in finding the activation times.

\section{Conclusion}

Noi-corr is superior to noi-iid when training set properties match those of the test beat. But if the confidence in the fidelity of the training set to the test data is low, noi-iid should be preferred, despite its amplitude attenuation effects. For applications requiring activation time derivation, careful selection of preprocessing methods, in particular to adequately remove high-frequency noise, is needed.

\section{Acknowledgements}

This is a collaborative work for the Consortium on ECG Imaging (CEI); all authors contributed equally. This work was supported in part by the French National Research Agency (ANR-10-IAHU04-LIRYC), and the grants VEGA 2/0071/16 and APVV-14-0875 in Slovakia.

\section{References}

[1] Cluitmans MJM, Peeters RLM, Westra RL, Volders PGA. Noninvasive reconstruction of cardiac electrical activity: update on current methods, applications and challenges. Neth Heart J 2015;23:301-311.

[2] Tikhonov AN, Arsenin VY. Solutions of Ill-posed Problems. Halsted Press, NY, 1977.

[3] Serinagaoglu Y, Brooks DH, MacLeod RS. Bayesian solutions and performance analysis in bioelectric inverse problems. IEEE Transactions on Biomedical Eng June 2005; 52(6):1009-1020.

[4] Aydin U, Serinagaoglu Y. A Kalman filter based approach to reduce the effects of geometric errors and the measurement noise in the inverse ECG problem. Med Biol Eng Comput 2011;49(9):1003-1013.
[5] Erem B, Coll-Font J, Orellana RM, Stóvicek P, Brooks DH. Using transmural regularization and dynamic modeling for noninvasive cardiac potential imaging of endocardial pacing with imprecise thoracic geometry. IEEE Transactions on Medical Imaging 2014;33(3):726-738.

[6] Zemzemi N, Dobrzynski C, Bear L, Potse M, Dallet C, Coudiere Y, Duboi R, Duchateau J. Effect of the torso conductivity heterogeneities on the ECGI inverse problem solution. In Computing in Cardiology. 2016; 233-236.

[7] Mazloumi Gavgani A, Serinagaoglu Dogrusoz Y. Noise reduction using $2 \mathrm{~d}$ anisotropic diffusion filter in inverse electrocardiography. In Mediterranean Conference on Medical and Biological Engineering and Computing. 2013; .

[8] Bear LR, Serinagaoglu Dogrusoz Y, Svehlikova J, CollFont J, Good W, van Dam E, MacLeod R, Abell E, Walton R, Coronel R, Dubois R. Effects of ECG signal processing on the inverse problem of electrocardiography. In Computing in Cardiology. 2018; .

[9] Stanley PC, Pilkington TC, Morrow MN. The effects of thoracic inhomogeneities on the relationship between epicardial and torso potentials. IEEE Trans Biomed Eng 1986; 33(3):273-284.

[10] Svehlikova J, Zelinka J, Serinagaoglu Dogrusoz Y, Good W, Tysler M, Bear LR. Impact of signal preprocessing on the inverse localization of the origin of ventricular tachycardia. In Computing in Cardiology. 2018; .

[11] Duchateau J, Potse M, Dubois R. Spatially coherent activation maps for electrocardiographic imaging. IEEE Transactions on Biomed Eng 2014;64(5):1149-1156.

Address for correspondence:

Dr. Y. Serinagaoglu Dogrusoz

Orta Dogu Teknik Universitesi

Elektrik and Elektronik Muh. Bol.

Ankara, Turkey

yserin@metu.edu.tr 